\title{
Stationary consistent equilibrium coalition structures constitute the recursive core*
}

\author{
László Á. Kóczy ${ }^{\dagger}$
}

\begin{abstract}
We study coalitional games where the coalitional payoffs depend on the embedding coalition structure. We introduce a noncooperative, sequential coalition formation model and show that the set of equilibrium outcomes coincides with the recursive core, a generalisation of the core to such games. In order to extend past results limited to totally recursive-balanced partition function form games we introduce a more permissive perfectness concept, subgame-consistency that only requires perfectness in selected subgames. Due to the externalities, the profitability of deviations depends on the partition formed by the remaining players: the stability of core payoff configurations is ensured by a combination of the pessimism of players going for certain profits only and the assumption that players base their stationary strategies
\end{abstract}

*The author thanks Lars Ehlers for his comments and the Hungarian Academy of Sciences for the financial support (LP-004/2010).

${ }^{\dagger}$ Centre for Economic and Regional Studies, Hungarian Academy of Sciences, Budaörsi út 45., H-1112 Budapest and Keleti Faculty of Business and Management, Óbuda University; koczy@krtk.mta.hu

The final publication is available at Elsevier via http://dx.doi.org/10.1016/j.jmateco.2015.08.006 
on a made-up history punishing some of the possible deviators - and getting this sometimes right.

Subject classification: $\mathrm{C} 71, \mathrm{C} 72$

Keywords and phrases: partition function, externalities, implementation, recursive core, stationary perfect equilibrium, time consistent equilibrium

\section{Introduction}

The classical theory of coalitional games studied games with orthogonal, isolated coalitions, that can be studied independently of each other. The characteristic function of a TU-game, for instance, assigns a payoff to a coalition disregarding other players and coalitions. For the usual interpretations of coalitions, be those trading blocks (Yi, 1996), trusts (Bloch, 1995) or international environmental agreements (Funaki and Yamato, 1999; Eyckmans and Tulkens, 2003), the orthogonality assumption is difficult to maintain; we believe it is the exception rather than the rule that coalitions can be studied independently of each other.

Since Thrall and Lucas (1963) introduced partition function form games numerous solution concepts have been proposed to solve cooperative games with externalities (Chander and Tulkens, 1995; Ray and Vohra, 1997; Hyndman and Ray, 2007). A solution concept is well-founded if it is fully characterised by a collection of elementary, usually independent properties, axioms or if it naturally emerges as the equilibrium of a noncooperative game. Few concepts for partition function form games have backing of either kind; for the axiomatic framework see for instance Bloch and van den Nouweland (2014), here we present an implementation by non-cooperative equilibria.

The implementation of cooperative solution concepts, such as the core in 
charancteristic function form games has an extensive literature (Chatterjee et al., 1993; Lagunoff, 1994; Perry and Reny, 1994), but these results do not directly generalise to games with externalities. In this domain Huang and Sjöström (2006) and Kóczy (2009) have provided partial results using sequential coalition formation games that have stationary perfect equilibria. Such games have non-empty cores in all subgames - a property that does not even hold for simple TU games. We therefore look for a condition that is weaker than subgame perfectenss. Subgame-consistency holds for a broader game class and the set of payoff-configurations generated by subgame-consistent strategies coincides with the recursive core.

Subgame-consistency is a weaker concept than subgame-perfectness, but more demanding than time-consistency (Kydland and Prescott, 1977). The latter insists only on the consistency of the equilibrium play, subgame-consistency also requires the perfectness of subgames that are relevant for the stability of the equilibrium, at last subgame-perfectness requires it in all subgames. Subgame-perfect equilibria are therefore also subgame-consistent and all subgame-consistent equilibria are also time-consistent. Moreover, stationary perfect equilibria are stationary consistent. For more on the relation of subgame-perfect and time-consistent strategies see Fershtman (1989) and Asilis (1995).

Unlike Huang and Sjöström (2006) and Kóczy (2009) we do not assume a fixed payoff division within coalitions, but allow an endogenous allocation. In a more recent paper Huang and Sjöström (2010) provide results for the r-core that closely correspond to ours, but the assumption that the game is totally r-balanced, that is, the $\mathrm{r}$-core of each residual subgame is non-empty remains. This difference turns out to be minor compared to the approach to handle a technical difficulty in the proofs. We explain these differences in 
the conclusion of the paper, after introducing our model.

The structure of the paper is as follows. After this introduction a long second section follows introducing both the cooperative and noncooperative theories to solve games in partition function form, we introduce the notation and simple terminology we are going to use. We present the cooperative solution, namely the recursive core and similarly the noncooperative coalition formation game and its equilibria. A novel equilibrium concept, subgame consistency and the corresponding notion of relevant subgame are also introduced here. We state and prove our main result in the third section. The paper ends with a brief conclusion.

\section{Preliminaries}

Let $N$ denote the set of players. Subsets are called coalitions. A partition $\mathcal{S}$ of $S$ is a splitting of $S$ into disjoint coalitions. $\Pi(S)$ denotes the set of partitions of $S$. In general we use capital and calligraphic letters to denote a set and its partition (the set of players $N$ being an exception), indexed capital letters are elements of the partition. We write $i \in \mathcal{S}$ if there exists $S_{k}$ such that $i \in S_{k} \in \mathcal{S}$ and if $i \in \mathcal{S}$ we write $\mathcal{S}(i)$ for the coalition embedded in $\mathcal{S}$ containing $i$.

The game $(N, V)$ is given by a player set $N$ and a partition function (Thrall and Lucas, 1963) $V: \Pi(N) \rightarrow\left(2^{N} \rightarrow \mathbb{R}\right)$, where $V\left(S_{i}, \mathcal{S}\right)$ denotes the payoff for coalition $S_{i}$ embedded in partition $\mathcal{S}$. In the examples we will abbreviate partitions; for instance we write $[1,23]$ for $\{\{1\},\{2,3\}\}$. We also write $V(\mathcal{P})=\left(V\left(S_{1}, \mathcal{P}\right), \ldots, V\left(S_{k}, \mathcal{P}\right)\right)$ if $\mathcal{P}=\left[S_{1}, \ldots, S_{k}\right]$ to denote the vector of coalitional payoffs embedded in the partition $\mathcal{P}$.

For vectors $x, y \in \mathbb{R}^{N}$ we write $x_{S}$ for the restriction to the set $S$ and 
$x_{S}>y_{S}$ if $x_{i} \geq y_{i}$ for all $i \in S \subset N$ and there exists $j \in S$ such that $x_{j}>y_{j}$.

Due to the externalities in the partition function form game the standard solution concepts do not work; we consider outcomes instead of imputations and the recursive core (Kóczy, 2007), a generalisation of the core, as the solution concept.

The pair $\omega=(x, \mathcal{P})$ consisting of a payoff vector $x \in \mathbb{R}^{N}$ and a partition $\mathcal{P} \in \Pi(N)$ is a payoff configuration (or outcome) if $\sum_{i \in S} x_{i}=V\left(P_{i}, \mathcal{P}\right)$ for all $P_{i} \in \mathcal{P}$. The set of outcomes of game $(N, V)$ is denoted $\Omega(N, V)$.

Let $S \subsetneq N$ and $\overline{\mathcal{S}}$ the partition of $\bar{S}=N \backslash S$. Then the residual game $S, V^{\overline{\mathcal{S}}}$ is the partition function game played over $S$ such that $V^{\overline{\mathcal{S}}}\left(S_{i}, \mathcal{S}\right)=$ $V\left(S_{i}, \mathcal{S} \cup \overline{\mathcal{S}}\right)$ for all $S_{i} \subseteq S$ and $\mathcal{S} \in \Pi(S)$.

Definition 1 (Recursive core (Kóczy, 2007)). For a single-player game the recursive core is trivially defined. Now assume that the core $C(N, V)$ has been defined for all games with $|N|<k$ players. Then for an $|N|$-player game an outcome $(x, \mathcal{P})$ is dominated if there exists a coalition $Q$ forming partition $\mathcal{Q}$ such that $y_{Q}>x_{Q}$ for all outcomes $(y, \mathcal{Q} \cup \overline{\mathcal{Q}}) \in \Omega(N, V)$ such that $\left(y_{\bar{Q}}, \overline{\mathcal{Q}}\right) \in C\left(\bar{Q}, V^{\mathcal{Q}}\right)$ if $C\left(\bar{Q}, V^{\mathcal{Q}}\right) \neq \varnothing$. The core $C(N, V)$ of $(N, V)$ is the set of undominated outcomes. 
Example 1. Consider the following 6-player partition function form game.

$$
\begin{aligned}
V([123456]) & =(12) \\
V([i, 123456 \backslash i]) & =(1,10) \quad \forall i \in N \\
V([i j, 123456 \backslash i j]) & =(2,4) \quad \forall i \neq j, i, j \in N \\
V([1, i, 23456 \backslash i]) & =(1,1,8) \quad \forall i \in N \backslash\{1\} \\
V([1,23,456]) & =(1,6,3) \\
V([1,23,4,56]) & =(0,6,1,2) \\
V([1,23,5,46]) & =(2,6,1,2) \\
V([1,23,6,45]) & =(3,2,1,2) \\
V([1,23,4,5,6]) & =(3,6,0,0,0)
\end{aligned}
$$

other payoffs are 0 .

A quick inspection of the partition function reveals that the (recursive) core consists of a single element $((2,2,2,2,2,2)$, [123456]). See Appendix A for the detailed calculations.

Example 2. Consider also a 4-player game $(\{1,2,3,4\}, U)$, where

$$
\begin{aligned}
U([1234]) & =(8) \\
U([i, j k l]) & =(1,5) \\
U([i j, k l]) & =(4,4) \\
U([i, j, k l]) & =(1,1,4) \\
U([1,2,3,4]) & =(1,1,1,1)
\end{aligned}
$$

if $\{i, j, k, l\}=\{1,2,3,4\}$.

A quick inspection of the partition function reveals that there are no deviations that could potentially give higher payoffs than under the payoff 
configuration $((1,1,1,1),[1234])$, while it can readily be verified that any pair can secure a (total) payoff of 4 .

Huang and Sjöström's (2003) r-core coincides with the recursive core on a broad class of games that does not, however include the standard TUgames without externalities. Ray's (2007) standard equilibrium, defined for symmetric partition function form games has a similar recursive structure. For an interpretation and the discussion of the properties of the recursive core see Kóczy (2007, 2009); Huang and Sjöström (2010).

\section{Sequential coalition formation}

The sequential coalition formation game we define is similar to Bloch's (1996) and Perry and Reny's (1994). First a player proposes the formation of some coalitions. The offer specifies not only who should be the members of these coalitions, but also how the coalitional payoffs will be shared. If all involved players accept the offer, the coalitions form and leave the game. When the offer is rejected, a new proposal is made and so on, until all players exit.

\subsection{The game}

Consider a game $(N, V)$ with a player set $N$ and partition function $V$. Time $t$ is continuous, but we assume that there is always an open time interval between two actions: there is time to respond. Let $Q^{t} \subseteq N$ denote those who have already quit the game by time $t$, forming partition $\mathcal{Q}^{t}$. Player $i$ can make proposals

$P_{i}^{t}=\left\{\left(\mathcal{P}^{t}, w^{t}\right) \mid \mathcal{P}^{t} \in \Pi\left(P^{t}\right), P^{t} \subseteq \overline{Q^{t}}, P^{t} \ni i, w^{t} \in \mathbb{R}^{P^{t}}, \forall P_{k}^{t} \in \mathcal{P}^{t} \sum_{j \in P_{k}^{t}} w_{j}^{t}=1\right\}$ 
the current proposer is $i^{t}$ making the proposal $p^{t}=\left(\mathcal{P}^{t}, w^{t}\right)$ to the players in $P^{t}$, already accepted by the players in $A^{t} \subseteq P^{t}$ (we assume $i^{t} \in A^{t}$ ) have already accepted the proposal. At $t$ a player $i$ can

1. accept the proposal $p^{t}$ if $i \in P^{t}$,

2. make a new proposal, or

3. do nothing.

The strategy $\sigma_{i}$ of a player $i$ specifies a complete protocol of actions for all times and contingencies during the game and $\sigma_{i}^{t}$ the action at $t$. Let $\sigma$ denote the strategy profile collecting the strategies of all players.

A state $s^{t}$ is a snapshot of the game and is given by a tuple $\left(\mathcal{Q}^{t}, i^{t}, p^{t}, A^{t}\right)$. History $h$ is a complete record of actions - we actually use only a small part of this information. Technically let $t_{1}, t_{2}, \ldots$ be points in time such that $s^{t_{k}}=s^{t} \neq s^{t_{k+1}}$ for all $t_{k}<t<t_{k+1}$. Then $h=\left\{s^{t_{k}}\right\}_{k}$. Let $h^{t}$ be a truncation of history up to time $t$. There is a one-to-one correspondence between history truncations and decision nodes in the game tree. We will identify subgames by truncated histories. Let $\left.\sigma\right|_{h^{t}}$ denote the restriction of strategy $\sigma$ to the subgame $h^{t}$.

There is no guarantee that the game ends, that all players quit. We therefore specify the payoffs for coalitions $Q_{k}$ embedded in an incomplete partition $\mathcal{Q}$. We take a conservative approach: players only receive their guaranteed payoff, the lowest value the coalition can obtain in a partition embedding the coalition structure of departed players:

$$
V\left(Q_{k}, \mathcal{Q}\right)= \begin{cases}\min _{\mathcal{P} \supset \mathcal{Q}} V\left(Q_{k}, \mathcal{P}\right) & Q_{k} \in \mathcal{Q} \\ 0 & \text { otherwise }\end{cases}
$$


For instance in Example 2 the departure of a singleton, say player 1 results in a simple majority residual game. For such games no stationary equilibria exist and so the bargaining will be inconclusive, but we can allocate the minimum achievable payoff $U(\{1\},[1])=\min _{\mathcal{P} \ni[1]}\{U(\{1\}, \mathcal{P})\}=1$ to this player - which is incidentally also the maximum, as in this case the payoff of this player does not depend on what happens in the rest of the game.

Given a strategy profile $\sigma$ the payoff of player $i$ is $x_{i}(\sigma)$.

\subsection{Equilibria}

Now that we have specified the available strategies (actions) and the resulting payoffs (incentives), we can focus on the outcomes of the coalition formation game. We hope to answer two questions simultaneously: (i) which coalitions will form (ii) how are coalitional payoffs distributed.

Recall that players are conservative and only go for certain profits: If different beliefs lead to different subsequent actions from the other players, a deviation may or may not be profitable under all such scenarios.

Definition 2. The strategy profile $\sigma^{*}$ is a subgame-perfect equilibrium if for all $h \in \mathcal{H}$, time $t, i \in N$, strategies $\sigma_{i}$ the corresponding restrictions $\left.\sigma^{*}\right|_{h^{t}}$ and $\left.\sigma_{i}\right|_{h^{t}}$ to the subgame at $h^{t}$ we have

$$
x_{i}\left(\left.\sigma^{*}\right|_{h^{t}}\right) \geq x_{i}\left(\left.\sigma_{i}\right|_{h^{t}},\left.\sigma_{-i}^{*}\right|_{h^{t}}\right) .
$$

The set of perfect equilibria may be too inclusive (see Muthoo (1990, 1995); Perry and Reny (1994); Osborne and Rubinstein (1990) for a discussion of folk-theorem-like results) so we focus on stationary strategies.

Definition 3. A strategy $\sigma$ is stationary if it does not depend on time. Formally: if for all $h$ and $t_{1}, t_{2}$ with $h^{t_{1}}=h^{t_{2}}$ we have $\left.\sigma\right|_{h^{t_{1}}}=\left.\sigma\right|_{h^{t_{2}}}$. 
Definition 4. A stationary perfect equilibrium $\sigma^{*}$ is a strategy profile that is both subgame-perfect and stationary.

For games with nonempty residual cores the set of stationary perfect equilibrium partitions coincide with the recursive core (Kóczy, 2009). This equivalence result predicts that games containing empty residual cores do not have stationary perfect equilibria.

Bloch (1996) presents a 3-player example, where player 1 would like to form a coalition with 2,2 with 3,3 with 1 . This game does not have stationary-perfect equilibria. Since residual games are also partition function form games, the smallest residual game for which the corresponding subgame of the sequential game has no stationary strategies has an empty core. By a sufficiently large payoff for the grand coalition the core of the original game is nevertheless empty. Perfectness only holds globally, that is, if the tiniest subgame fails to have stationary perfect equilibria this failure extends to the entire game. On the other hand, just as the recursive core may be non-empty even if the game has empty residual cores, with a weaker concept of perfection we may retain an essentially perfect behaviour in the corresponding sequential coalition formation games, too.

Time-consistency (Kydland and Prescott, 1977) requires the equilibrium strategy to be consistent or revision-free and is therefore unaffected by empty cores elsewhere. Most subgames are never reached anyway so following this promising direction for weakening subgame perfectness we are focussing on the immediate neighbourhood of the equilibrium play $\sigma$. A strategy profile is in this immediate neigbourhood if it is an elementary deviation from $\sigma$, that is, it only differs in the action of a single player in a single decision node. Subgame-consistency, that we introduce below, is such that the perfectness/consistency criterion is only required in relevant subgames. What 
is relevant?

Definition 5. For a strategy profile $\sigma$ a subgame at $t$ is relevant if

1. it is the original game $(t=0)$,

2. it can be reached via an elementary profitable deviation from $\sigma$, or

3. it is a relevant subgame of a relevant subgame.

Let $\left.\sigma\right|_{h}$ denote the truncation of $\sigma$ to the subgame corresponding to $h$.

Definition 6. The strategy profile $\sigma^{*}$ is a subgame-consistent equilibrium if for all relevant subgames $h^{t}, i \in N$, strategies $\sigma_{i}$ the corresponding restrictions $\left.\sigma^{*}\right|_{h^{t}}$ and $\left.\sigma_{i}\right|_{h^{t}}$ to $h^{t}$ we have

$$
x_{i}\left(\left.\sigma^{*}\right|_{h^{t}}\right) \geq x_{i}\left(\left.\sigma_{i}\right|_{h^{t}},\left.\sigma_{-i}^{*}\right|_{h^{t}}\right) .
$$

For an equilibrium strategy profile, this requires checking the equilibrium path only: Since no profitable deviation exist, other subgames need not be checked. Were there profitable deviations they would have to be supported by a strategy that is subgame perfect along that strategy. The equilibrium in Example 2 is to propose and accept the core payoff configuration. We must of course check possible deviations, but if a particular deviation cannot possibly be profitable irrespective of what happens in the rest of the game, it is actually unnecessary to check what would happen there. As such, since player 1 can never get more than 2 , so there is no need to evaluate the (problematic) subgame induced by its departure.

Clearly, subgame perfect equilibrium strategies are also subgame-consistent.

\subsection{Alternative histories}

For a general implementation result we seek equilibrium strategy profiles that produce core outcomes. In Subsection 3.2 we have solved the issue due 


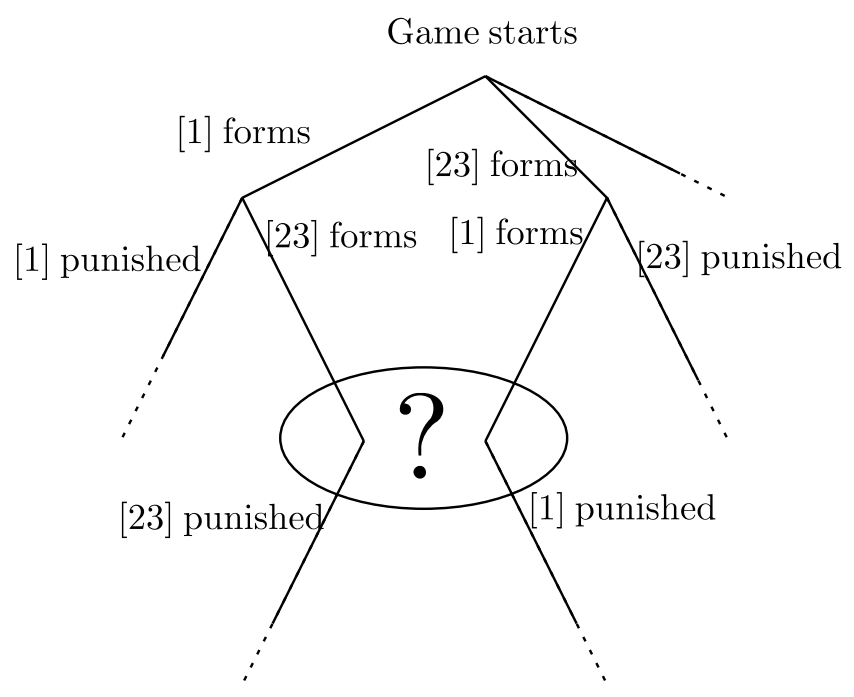

Figure 1: Stationary strategies cannot react to different histories.

to empty residual cores, here we describe and solve another problem due to stationarity.

Consider Example 1 again. As we have seen it, in equilibrium players get a payoff of 2 each. We, however, consider the scenario when the partition $[1,23]$ has already been formed. What is the strategy for the remaining players and in particular: what will be the payoff of 1,2 , and 3 ? In the cooperative game the reaction depends on the history that has led to this state. If [1] deviated first, then in the induced subgame players 2 to 5 should have formed the "grand coalition" in this game. This would give player 1 a payoff of 1 that would make the deviation non-profitable. In this smaller grand coalition players 2 and 3 would get a payoff of 2 each and leave the game prematurely only if they are sure to obtain more. In the cooperative game their departure is followed by the formation of a residual payoff configuration; here coalition $\{2,3\}$ fears that the partition $[45,6]$ emerges giving a lower payoff than in the residual grand coalition. Since the core includes payoff configurations with 
this partition, it would make sense for the players 4,5 and 6 to choose this strategy. Except that there is another possibility. Perhaps it is 2 and 3 that said first no to the original proposal and left. The equilibrium answer was then subsequently sabotaged by 1 , who would have a payoff of 1 , but now hopes to get 3 . We are, again in the same subgame, but in the cooperative game we now look at residual core-supporting partitions that are bad for [1], such as $[4,56]$. Note that $[45,6]$, what was the only good partition before, does not work here. For the last deviation by $\{1,2,3\}$ to form partition $[1,23]$ either of the two strategies would "work", but otherwise the equilibrium strategy seems to depend on the history.

When looking at the sequential game we must explicitly determine which equilibrium strategy responds to a particular history, which equilibrium strategy can render the same deviations non-profitable. When looking at stationary strategies, history is masked from the players, who only see the current state of the game. At some point players may see a state that is very different from the equilibrium play. What is the equilibrium that prevents such deviations?

Before elaborating on this issue we must stress that in the sequential coalition formation game players do not "respond" to formed coalitions and especially not punish those. On the other hand there may be different strategies that constitute equilibrium behaviour in a given subgame and we are interested in those that contribute to stability in the larger game, too.

Although players do not know the history, given the current state $s$ they can reconstruct one of the possible histories $h(s)$ satisfying $s^{h^{t}(s)}=s$ for some $t$. This history $h(s)$ provides a plausible, but not necessarily true explanation of the current state. Let $\mathcal{H}(s)=\left\{h \mid \exists t: s^{h^{t}}=s\right\}$ be the set of plausible histories to the current state $s$. 


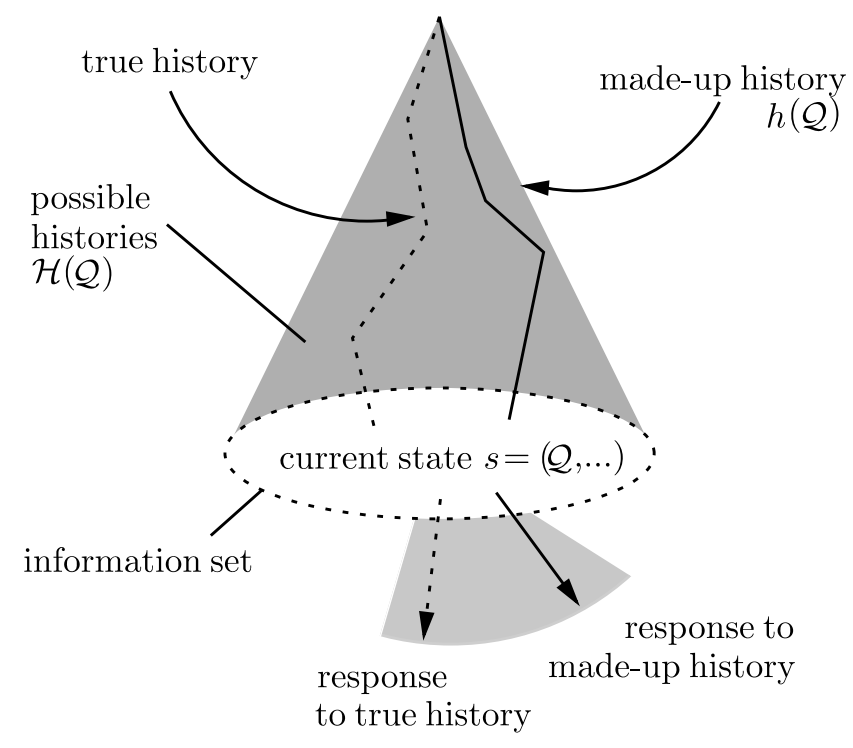

Figure 2: Stationary decision with possible histories

In Example 1 the existing partition [1,23] is possible with - essentially three different histories. Since 4, 5 and 6 do not know which of these is the true one, they make up a history and follow the strategy conditional on that.

Unfortunately in a stationary process these possible histories only provide a temporary explanation as they get forgotten, too. We assume that $h$ is arbitrarily regenerated when the partition $\mathcal{Q}^{t}$ changes, that is, each time some players quit the game. It is common knowledge among the remaining players, who choose their strategies treating $h$ as the true history, but taking into account that future deviations may generate a new alternative history. "History" is not preserved, subsequent alternative histories are totally unrelated, it may well be that the current state will not have happened at all. Let $\mathcal{H}(\sigma)$ denote the set of all possible histories happening as $\sigma$ is played (and there are no deviations). Finally $\mathcal{H}(\sigma, s) \subseteq \mathcal{H}(\sigma) \cap \mathcal{H}(s)$ denotes the set of possible histories passing through $s$ when $\sigma$ is played after $s$ is reached. Let us stress that many of the $h \in \mathcal{H}(\sigma, s)$ will believe that $s$ did not exist at all, 
but at $s$ players are aware of their limited rationality in the future.

The coalitional payoffs are not related to history and are thus unaffected, but what happens to the payoff of the individual players? In the non-stationary game players can calculate the payoffs by just looking at the strategies. With stationarity we must take the subsequent updating of the history into account. The subsequent development of the game therefore depends on the current state $s$ - in particular, the partition $\mathcal{Q}$ - and the strategy restricted to this subgame. Since we focus on stationary strategies it is sufficient to say that these strategies are restricted to a subgame $s$. Then the subsequent development of history depends on some strategy $\left.\sigma\right|_{s}$ restricted to $s$. Let $x(\sigma, h) \in \mathbb{R}^{N}$ denote the vector of payoffs in case $\sigma$ is played along the history $h$. Then the payoff players can expect is

$$
x(\sigma, s)=\min _{h \in \mathcal{H}(\sigma, s)} x(\sigma, h) .
$$

Note the pessimism of the players. When uncertain about the subsequent development of the game, they assume that the remaining players will fabricate histories that are the least favourable to them. While subgame perfectnes can be formulated with these expectations, too, the resulting equilibria are different in general. Since the additional "information" comes from the past, the concepts of stationarity and stationary equilibria are not affected, the stationary equilibria remain the same and the recursive core equivalence result remains valid. Likewise, subgame consistency can be redefined in this environment, but we first clarify what is a relevant subgame.

Definition 7. For a strategy profile $\sigma$ a subgame at $h^{t}$ is relevant if

1. it is the original game $\left(\mathcal{Q}^{h^{t}}=\varnothing\right)$,

2. there exists an elementary deviation $\sigma^{\prime}$ producing $\mathcal{Q}^{\prime}$ such that

$$
x_{i}\left(\sigma, s^{h^{t}}\right)<x_{i}\left(\sigma^{\prime}, \mathcal{Q}^{\prime}, \varnothing\right),
$$


3. or it is a relevant subgame of a relevant subgame.

The subgame-consistency is accordingly modified replacing $h$ by an arbitrary (compatible) history $h(s)$ in Inequality 3.3 and payoffs are now given by Equation 3.4 and are conditional on the current $s$ via the different updates of made-up history.

$$
x_{i}\left(\left.\sigma^{*}\right|_{h^{t}(s)}, s\right) \geq x_{i}\left(\left.\sigma_{i}\right|_{h^{t}(s)},\left.\sigma_{-i}^{*}\right|_{h^{t}(s)}, s\right) .
$$

The condition becomes clear now: it has implications not so much for the present, but for the reactions of the remaining players.

Let $\sigma$ be a stationary strategy and $\left.\sigma\right|_{s}$ its restriction to a state $s$.

A stationary consistent equilibrium $\sigma^{*}$ is a strategy profile that is both subgame-consistent and stationary, that is, if for all relevant subgames corresponding to some $s$ we have

$$
x_{i}\left(\left.\sigma^{*}\right|_{s}, s\right) \geq x_{i}\left(\left.\sigma_{i}\right|_{s},\left.\sigma_{-i}^{*}\right|_{s}, s\right) \text {. }
$$

We denote the set of stationary consistent equilibria by $\operatorname{SCE}(N, V)$ and outcomes resulting from playing such equilibrium strategies by $\Omega^{*}(N, V)$.

\section{Results}

Theorem 1. Let $(N, V)$ be a partition function form game. Then its recursive core $C(N, V)$ coincides with the set $\Omega^{*}(N, V)$ of outcomes supported by stationary consistent equilibrium strategy profiles.

The rest of this section is devoted to the inductive proof of this theorem. As the proof is long, we break it into a number of propositions and finally present a summary of these results. The first proposition requires no proof: 
Proposition 2. Let $(\{1\}, V)$ be a trivial, single-player partition function form game. Then $C(\{1\}, V)=\Omega^{*}(\{1\}, V)$.

Now assume that Theorem 1 holds for all games with less than $k$ players. In the following we extend it to games with $k$ players. In order to show $\Omega^{*}(N, V)=C(N, V)$, first we show $\Omega^{*}(N, V) \subseteq C(N, V)$ then $\Omega^{*}(N, V) \supseteq$ $C(N, V)$.

Lemma 3. If Theorem 1 holds for all games with up to $k-1$ players, $\Omega^{*}(N, V) \subseteq C(N, V)$ for all $k$-player games.

Proof. If $\Omega^{*}(N, V)=\varnothing$ the result is trivial, otherwise there exists a SCE $\sigma$ producing $\omega(\sigma, h)=(x(\sigma, h), \mathcal{P}(\sigma, h)) \in \Omega^{*}(N, V)$ for some sequence of possible histories $h \in \mathcal{H}(\sigma, \varnothing)$. In particular, we assume that $\omega(\sigma, h) \notin$ $C(N, V)$ and prove contradiction.

By this assumption there exists a profitable deviation $\mathcal{D}$ by some set $D$ of players. By this we really mean a deviation by a single player $i \in D$ that results in the departure of $\mathcal{D}$, that is, in a state $s^{\prime}$ with $\mathcal{Q}^{s^{\prime}}=\mathcal{D}$, where, without loss of generality, we assume that the deviation occurs at $s$ when no other players have yet left the game. The induced subgame has fewer players so the inductive assumption can be applied. In the sequential game the deviation at $h^{t}$ is expressed by the strategy profile $\sigma^{\prime}=\left(\left.\sigma_{i}\right|_{-h^{t}},\left.\sigma_{i}^{\prime}\right|_{h^{t}}, \sigma_{-i}\right)$ differing only for $i$ and only in the subgame $h^{t}$. We discuss three cases.

Case 1. For the strategy profile $\sigma^{\prime}$ the subgame at $\mathcal{D}$ is not relevant. Then for all $\sigma_{-i}$ there exists $i \in D$ and $h^{\prime} \in \mathcal{H}\left(\sigma^{\prime}, s^{\prime}\right)$ such that $x_{i}\left(\sigma^{\prime}, h^{\prime}\right)<$ $x_{i}(\sigma, h)$ - thus the deviation cannot be profitable in the cooperative game; contradiction.

Case 2. The subgame is relevant, the core of the corresponding residual 
subgame is empty. Then $V(D, \mathcal{D} \cup \overline{\mathcal{D}})>\sum_{i \in S} x_{i}(\sigma, h)$ for all $\overline{\mathcal{D}} \in \Pi(\bar{D})$. As

$$
V(D, \mathcal{D} \cup \overline{\mathcal{D}})=\min _{h^{\prime} \in \mathcal{H}\left(\sigma^{\prime}, \mathcal{D}\right)} \sum_{i \in S} x_{i}\left(\sigma^{\prime}, h^{\prime}\right)
$$

a player $i$ in $D$ should immediately propose to form $\mathcal{D}$. By subgame consistency all in $D$ will accept. Therefore $\sigma$ is not a stationary consistent equilibrium, moreover the outcome $\omega(\sigma, h)$ cannot be supported by other equilibria either. Contradiction.

Case 3. The induced subgame is relevant and the core of the corresponding residual subgame is not empty. Since $\sigma$ is a SCE its restriction $\left.\sigma\right|_{s}$ to this relevant subgame $s$ is stationary consistent, too. Moreover the deviation from $\sigma$ to form $\mathcal{D}$ is not profitable, therefore

$$
x_{D}\left(\left.\sigma\right|_{s}, s\right) \geq x_{D}\left(\left.\sigma^{\prime}\right|_{s^{\prime}}, s^{\prime}\right)
$$

On the other hand, by the inductive assumption,

$$
\omega\left(\left.\sigma^{\prime}\right|_{s^{\prime}}, s^{\prime}\right) \in C\left(\bar{D}, V^{\mathcal{D}}\right)
$$

This, however, implies that the deviation $\mathcal{D}$ is not profitable in the cooperative game; contradiction.

We have discussed all cases, and found the assumptions contradicting. Therefore $\omega(\sigma, H) \in C(N, V)$.

Punishment strategy Before we move on to our next lemma, we specify a "response" to each deviation that turns these deviations unprofitable. In the recursive core a deviation is only profitable if it represents an improvement in the payoffs for all residual assumptions. In a game $(N, V)$ the core is nonempty if for all outcomes $(x, \mathcal{P}) \in C(N, V)$ and for all deviations $\mathcal{D}$ there exists an outcome $\left(y_{\bar{D}}, \overline{\mathcal{D}}\right) \in \Omega\left(\bar{D}, V^{\mathcal{D}}\right)$ such that 
1. there exists $D_{1} \in \mathcal{D}$ such that $\sum_{i \in D_{1}} x_{i} \geq V\left(D_{1}, \mathcal{D} \cup \overline{\mathcal{D}}\right)$ and

2. $\left(y_{\bar{D}}, \overline{\mathcal{D}}\right) \in C\left(\bar{D}, V^{\mathcal{D}}\right)$ if $C\left(\bar{D}, V^{\mathcal{D}}\right) \neq \varnothing$.

Generally, for a residual game $\left(\bar{S}, V^{\mathcal{S}}\right)$ the response to $\mathcal{D}$ is denoted as $\left(x_{\bar{S}}(\mathcal{D}), \overline{\mathcal{S}}(\mathcal{D})\right)$.

In the sequential game, there may be equilibrium strategies for the subgame that make the deviation $\mathcal{D}$ unattractive. Such strategies deserve special attention as these help to preserve the equilibrium in the greater game. We call these strategies "punishment strategies" although - recall - these players do not react to the deviation, as such, but simply play an equilibrium that works. Note that finding the right punishment can be quite difficult.

In the following we specify the punishment strategy to a deviation knowing that some other coalitions left, too. We assume that $\mathcal{Q}$ has already left the game, but $\tilde{\mathcal{Q}} \subseteq \mathcal{Q}$ was (or at least $\bar{Q}$ think it was) the last to exit. In the partition function form game $\left(\overline{Q \backslash \tilde{Q}}, V^{\mathcal{Q} \backslash \tilde{\mathcal{Q}}}\right)$ the partition $\tilde{\mathcal{Q}}$, as a deviation, defines a residual game $\left(\bar{Q}, V^{\mathcal{Q}}\right)$, where the response to $\tilde{\mathcal{Q}}$ is $\left(x_{\bar{Q}}(\tilde{\mathcal{Q}}), \overline{\mathcal{Q}}(\tilde{\mathcal{Q}})\right)$.

Lemma 4. If Theorem 1 holds for all games with less than $k$ players, then $\Omega^{*}(N, V) \supseteq C(N, V)$ for all $k$-player games $(N, V)$.

Proof. The proof is partly inspired by Bloch's (1996, Proposition 3.2), and is by construction. We show that if $\left(x^{*}, \mathcal{P}^{*}\right) \in C(N, V)$ there exists a stationary consistent strategy profile $\sigma^{*}$ such that for all for all possible histories $h \in$ $\mathcal{H}\left(\sigma^{*}, \varnothing\right)$ we have $\omega\left(\sigma^{*}, h\right) \in C(N, V)$.

In the following we define the stationary strategy $\sigma_{i}^{*}$ for player $i$. Due to stationarity it is sufficient to specify the strategy for each triple $(\mathcal{Q}, \tilde{\mathcal{Q}}, p)$ consisting of the partition of players who have already quit, the subpartition consisting of the coalitions that left last according to the current made-up 
history and the current proposal $p=(\mathcal{T}, w)$. Then the strategy of player $i$ is given as

$\sigma_{i}^{*}(\mathcal{Q}, \tilde{\mathcal{Q}}, \mathcal{T}, w)= \begin{cases}\text { accept } & \text { if } x_{i}\left(\sigma^{*}, \mathcal{Q} \cup \mathcal{T}, \varnothing\right)>x_{i}\left(\sigma^{*}, \mathcal{Q}, \varnothing\right) \\ \left(\mathcal{P}^{*}, \frac{x *}{\left|x^{*}\right|}\right) & \text { if } \mathcal{T}=\mathcal{Q}=\varnothing \\ \left(\overline{\mathcal{Q}}(\tilde{\mathcal{Q}}), \frac{x_{\overline{\mathcal{Q}}}(\tilde{\mathcal{Q}})}{\left|x_{\bar{Q}}(\tilde{\mathcal{Q}})\right|}\right) & \text { if } \mathcal{T}=\varnothing, \text { but } \mathcal{Q} \neq \varnothing \\ \text { wait } & \text { otherwise. }\end{cases}$

In equilibrium $\mathcal{P}\left(\sigma^{*}\right)=\mathcal{P}^{*}$ and the strategy is stationary by construction so we only need to verify subgame-consistency. We show this by induction. As subgame-consistency holds for a trivial game we may assume that it holds for all games of size less than $|N|$.

Now consider game $(N, V)$ and observe that if $Q$ departed to form $\mathcal{Q}$ the subgame is simply a coalition formation game with less players. We discuss two cases based on the emptiness of the residual core.

1. If the residual core is not empty, the proposed strategy exhibits the same similarity property: in equilibrium the core partition is proposed and accepted, while residual cores form off-equilibrium.

The inductive assumption then ensures that the off-equilibrium path is subgame-consistent so we only need to check whether a deviation $\tilde{\mathcal{Q}}$ is ever accepted. This deviation corresponds to a deviation in the partition function game. Since $\left(x^{*}, \mathcal{P}^{*}\right) \in C(N, V)$, by the construction of $\left(\overline{\mathcal{Q}}(\tilde{\mathcal{Q}}), \frac{x_{\bar{Q}}(\tilde{\mathcal{Q}})}{\left|x_{\bar{Q}}(\tilde{\mathcal{Q}})\right|}\right)$ we know that for some history $h(\mathcal{Q})$ there exists a player in $\tilde{Q}$ for whom the deviation $\tilde{\mathcal{Q}}$ is not profitable. Given the pessimism of the players, this is sufficient to deter this player from accepting the proposal to deviate.

2. The emptiness of the residual core, by our assumption, implies that 
there are no stationary consistent equilibrium strategy profiles. In the absence of such strategy profiles the strategy $\sigma^{*}$ will be abandoned and so the players in $\tilde{Q}$ cannot predict the partition of $\overline{\mathcal{Q}}$ - in this case, by Expression 3.1, they, individually, expect the worst. As $\tilde{\mathcal{Q}}$ only forms if it is a profitable deviation, that is, only if $x_{i}\left(\sigma^{*}, h\right)$ is an improvement for all $h \in \mathcal{H}\left(\sigma^{*}, \mathcal{Q}, \varnothing\right)$. Since $\left(x^{*}, \mathcal{P}^{*}\right) \in C(N, V)$ this is not the case. This, implies that post-deviation subgame is not relevant. Also, the formation of $\mathcal{P}^{*}$ is unaffected by possible deviations in this subgame, meeting the first condition of subgame-consistency.

Proof of Theorem 1. The proof is by induction. The result holds for trivial, single-player games. Assuming that the result holds for all $k-1$ player games, the result for $k$-player games is a corollary of Lemmata $3 \& 4$.

\section{Conclusion}

Theorem 1 holds for arbitrary games in discrete partition function form, but of course it is most interesting for games where some of the residual cores contain payoff configurations with different partitions or are empty. When a proposal is made in a game without externalities the invited players do not even (need to) consider the residual game and therefore the emptiness of a residual core is not addressed. Huang and Sjöström (2006) and Kóczy (2009) focus on games where the residual cores are non-empty, in fact the r-core (Huang and Sjöström, 2003) is not even defined for games with empty residual cores. As already pointed out by Kóczy (2007) this is not only an enormous limitation given the number of conditions such games must satisfy (one for each residual game), but the definitions/results do not apply to some games without externalities and so they are not generalisations of the 
well-known results for TU-games. The present paper heals this deficiency.

We have already indicated in our introduction the similarity with the results by Huang and Sjöström (2010). While Huang and Sjöström (2010) insist on totally r-balanced games, the main conceptual difference seems to be the way alternative histories are handled. Their solution is admittedly elegant and simple: the process "remembers" the coalition that deviated last. When subgame perfect equilibria exist, we can basically say that the remaining players can select a retaliation to punish this coalition. In contrast we insist on the memoryless nature of the process: no information on past actions is kept, only the result of the actions. Instead, players have a common understanding of what may have happened in the past and react to that. Given the conservativism of the players it is enough if the players in the subgame get it right occasionally. This approach is more in line with the recursive definition of the recursive core, where the residual game is a game on its own: influenced by the deviating coalitions, but played independently of these.

If the concept is a generalisation of the core for TU-games, it is natural to ask how our game proceeds in the special case when the partition function form game at hand is actually a TU-game as it does not have externalities. In the absence of externalities, there is no way to "punish" deviating coalitions as their payoff does not depend on the partition of the remaining players. Since there are no punishments, any strategy profile resulting in a core outcome in the remaining game will equally be a punishment strategy and therefore considering alternative histories does not really have a bite here.

Similarly, the expectation that the residual players will form a residual core outcome does not influence the decisions of active players: their payoff 
will not depend on the coalitions formed in the residual game. Perry and Reny (1994) study market games (Shapley and Shubik, 1969), which are totally-balanced. For such games the "residual core" is always non-empty and therefore $i$ ) the game always terminates with all players leaving the game and ii) there always exist stationary perfect equilibria. For such games subgame consistency and subgame perfectness coincide and so the two procedures provide the same implementation.

\section{A The core of the game in Example 1}

In the following we present the calculations to determine the core of the game in Example 1.

Firstly note that the game is cohesive: the payoff of the grand coalition is strictly the highest among all partitions and therefore all core payoffconfigurations must have the grand coalition as partition. It is also clear that no player can have a payoff greater than 2 as any five players can obtain 10 by deviating - in this case the residual game is trivial - and therefore all players must have a payoff of 2 exactly. Therefore the core is a subset of $\{((2,2,2,2,2,2),[123456])\}$, are there other deviations that would make it empty? In the following we look at all deviations that may be profitable.

Consider a deviation by player 1 . In order to determine its payoff we must first evaluate the induced residual game This deviation leads to the following residual game: 


$$
\begin{aligned}
V^{[1]}([23456]) & =(10) \\
V^{[1]}([i, 23456 \backslash i]) & =(1,8) \quad \forall i \in N \backslash\{1\} \\
V^{[1]}([23,456]) & =(6,3) \\
V^{[1]}([23,4,56]) & =(6,1,2) \\
V^{[1]}([23,5,46]) & =(6,1,2) \\
V^{[1]}([23,6,45]) & =(2,1,2) \\
V^{[1]}([23,4,5,6]) & =(6,0,0,0)
\end{aligned}
$$

other payoffs are 0 .

This game is also cohesive and any four players can get 8 , so the candidate for the residual core is $\{((2,2,2,2,2),[23456])\}$. No single-player deviation can be profitable, however a deviation by $\{2,3\}$ can lead to a payoff of 2 or 6 depending on what happens in the induced residual game.

$$
\begin{aligned}
V^{[1,23]}([456]) & =(3) \\
V^{[1,23]}([i, j k]) & =(1,2) \quad \forall\{i, j, k\}=\{4,5,6\} \\
V^{[1,23]}([4,5,6]) & =(0,0,0) .
\end{aligned}
$$

This game is also cohesive, but not strictly. Any pair can obtain a payoff of 2 , but not more. It is easy to verify that the core consists of the following payoff configurations

$$
\{((1,1,1),[456]),((1,1,1),[4,56]),((1,1,1),[5,46]),((1,1,1),[6,45])\} .
$$

So what does this imply for $\{2,3\}$ ? Their initial restricted payoff vector is $(2,2)$ and the payoff of their coalition is $6,6,6,2$ respectively for the 
different residual core partitions. The recursive core assumes that players are pessimistic: unless the deviation is profitable under all circumstances it does not go through. Note that no other deviation promises to be profitable, so $\{((2,2,2,2,2),[23456])\}$ is undominated. Then the payoff of 1 in the primary deviation is 1 and therefore the deviation is not profitable.

We do not need to look at residual subgames where the deviating coalition can never have a payoff higher than the total payoff obtained under the core (that is: 2). This includes all other singletons and in fact all other coalitions except for the pair [23].

A deviation by [23] gives

$$
\begin{aligned}
V^{[23]}([1456]) & =(4) \\
V^{[23]}([1,456]) & =(1,3) \\
V^{[23]}([1,4,56]) & =(0,1,2) \\
V^{[23]}([1,5,46]) & =(2,1,2) \\
V^{[23]}([1,6,45]) & =(3,1,2) \\
V^{[23]}([1,4,5,6]) & =(3,0,0,0)
\end{aligned}
$$

other payoffs are 0 . We show that the core includes $\{((1,1,1,1),[1456])$, $((1,1,1,1),[1,456]),((0,1,1,1),[1,4,56]),((2,1,1,1),[1,5,46]),((3,1,1,1),[1,6,45])\}$. The only deviation that could challenge such outcomes is by the singleton [1]. (We make the - somewhat debatable - assumption that a subset of the current partition may also deviate.). We have already studied the induced subgame $V^{[1,23]}$ above and found that the core consists of the following payoff configurations $\{((1,1,1),[456]),((1,1,1),[4,56]),((1,1,1),[5,46]),((1,1,1),[6,45])\}$. A pessimistic player 1 will then expect a payoff of 0 that is no improvement.

At last we must also look for coalitions that deviate by forming a non-trivial - partition. Here the deviation is profitable even if only one 
of the coalitions has a higher total payoff as long as none of the other coalitions loose payoff. We only need to look at the coalition $\{1,2,3\}$ forming a partition $[1,23]$. We have already looked at the induced residual game $\left(\{4,5,6\}, V^{[1,23]}\right)$, found that (1) the core is not empty, and (2) for some core elements the deviation is unworthy therefore, under pessimism the deviation is not profitable.

\section{References}

Asilis, C. M., 1995, A note on the equivalence of time consistency and subgame perfection in stochastic games, European Economic Review 39, 245251.

Bloch, F., 1995, Endogenous structures of association in oligopolies, RAND Journal of Economics 26, 537-556.

Bloch, F., 1996, Sequential Formation of Coalitions in Games with Externalities and Fixed Payoff Division, Games and Economic Behavior 14, 90-123.

Bloch, F. and A. van den Nouweland, 2014, Expectation formation rules and the core of partition function, Games and Economic Behavior 88, 339-353.

Chander, P. and H. Tulkens, 1995, A core-theoretic solution for the design of cooperative agreements on transfrontier pollution, International Tax and Public Finance 2, 279-293.

Chatterjee, K., B. Dutta, D. Ray, and K. Sengupta, 1993, A Noncooperative Theory of Coalitional Bargaining, Review of Economic Studies 60, 463477. 
Eyckmans, J. and H. Tulkens, 2003, Simulating coalitionally stable burden sharing agreements for the climate change problem, Resource and Energy Economics 25, 299-327.

Fershtman, C., 1989, Fixed rules and decision rules:: Time consistency and subgame perfection, Economics Letters 30, 191-194.

Funaki, Y. and T. Yamato, 1999, The core of an economy with a common pool resource: A partition function form approach, International Journal of Game Theory 28, 157-171.

Huang, C.-Y. and T. Sjöström, 2003, Consistent solutions for cooperative games with externalities, Games and Economic Behavior 43, 196-213.

Huang, C.-Y. and T. Sjöström, 2006, Implementation of the recursive core for partition function form games, Journal of Mathematical Economics 42, $771-793$.

Huang, C.-Y. and T. Sjöström, 2010, The Recursive Core for NonSuperadditive Games, Games 1, 66-88.

Hyndman, K. and D. Ray, 2007, Coalition Formation with Binding Agreements, Review of Economic Studies 74, 1125-1147.

Kóczy, L. Á., 2007, A recursive core for partition function form games, Theory and Decision $63,41-51$.

Kóczy, L. Á., 2009, Sequential Coalition Formation and the Core in the Presence of Externalities, Games and Economic Behavior 66, 559-565.

Kydland, F. E. and E. C. Prescott, 1977, Rules Rather than Discretion: The Inconsistency of Optimal Plans, Journal of Political Economy 85, 473-491. 
Lagunoff, R. D., 1994, A simple noncooperative core story, Games and Economic Behavior 7, 51-61.

Muthoo, A., 1990, Bargaining without commitment, Games and Economic Behavior 2, 291-297.

Muthoo, A., 1995, A bargaining model with players' perceptions on the retractability of offers, Theory and Decision 38, 85-98.

Osborne, M. J. and A. Rubinstein, 1990, Bargaining and markets, vol. 59 (Academic Press, San Diego).

Perry, M. and P. J. Reny, 1994, A noncooperative View of Coalition Formation and the Core, Econometrica 62, 795-817.

Ray, D., 2007, A Game-Theoretic Perspective on Coalition Formation (Oxford University Press, Oxford).

Ray, D. and R. Vohra, 1997, Equilibrium Binding Agreements, Journal of Economic Theory 73, 30-78.

Shapley, L. S. and M. Shubik, 1969, On market games, Journal of Economic Theory 1, 9-25.

Thrall, R. M. and W. F. Lucas, 1963, N-Person Games in Partition Function Form, Naval Research Logistics Quarterly 10, 281-298.

Yi, S.-S., 1996, Endogenous formation of customs unions under imperfect competition: open regionalism is good, Journal of International Economics 41, 153-177. 